\title{
Landau level shift under the influence of short-range impurities in gapless graphene
}

\author{
J. Munárriz and F. Domínguez-Adame \\ GISC, Departamento de Física de Materiales, Universidad Complutense, E-28040 \\ Madrid, Spain \\ E-mail: j.munarriz@fis.ucm.es
}

\begin{abstract}
.
We study theoretically the level shift of massless Dirac fermions in a graphene monolayer subjected to a quantizing perpendicular magnetic field under the influence of short-range impurities. A Green function method is used to obtain closed expressions for the Landau level shift for any sharply peaked impurity potential approaching a $\delta$ shell potential.

PACS numbers: 81.05.ue, 03.65.Pm, 71.70.Di
\end{abstract}

Journal of Physics A: Mathematical and Theoretical 45, 305002 (2012) 


\section{Introduction}

Electron properties in graphene have been an active research topic since the experimental realization of a single layer of carbon atoms arranged in a honeycomb lattice $[1,2]$. It is now well-established that low-energy electronic excitations in pristine monolayers of graphene can be described by the massless $(2+1)$ Dirac equation [3, 4]. In addition, breaking of sublattice symmetry owing to the interaction with a substrate may open a gap in epitaxial graphene [5]. The gap enters as a mass term in the Dirac equation, usually referred to as scalar potential [6]. Thus, graphene provides an interesting realization of massless and massive quantum electrodynamics in two dimensions [7].

The discovery of the integer $[1,8,9]$ and fractional $[10,11]$ quantum Hall effects has spurred the interest on electron transport in graphene under strong magnetic fields. The transition from linear or parabolic bands in graphene to Landau levels as the magnetic field is increased can be successfully studied using a tight-binding model for the $\pi$ orbitals [12]. This transition has also been observed in graphene quantum dots, as reported in Ref. [13]. In this work it was concluded that the details of the transition are strongly affected by the defects of the underlying graphene lattice, among other effects. The dependence of the main features of the transition on the imperfections of graphene prompts the development of the scattering theory for Dirac fermions by point defects under quantizing magnetic field, namely in the Landau level regime.

Our aim in this paper is to study the Landau level shift by the presence of a shortrange impurity in graphene. In this context, short-range impurity refers to the absence of long-range tails in the interaction potential [14]. Bound electron states in monolayers of gapped graphene with short-range defects have already been discussed by Firsova et al. in Ref. [14]. These authors replaced the actual impurity potential of the types local chemical potential and local gap by vector (the time component of a Lorentz vector) and scalar $\delta$-shell functions, respectively. Bound states of the $(2+1)$ Dirac equation with a vector-like $\delta$-shell potential were also earlier discussed by Dong et al. in Ref. [15] (there is a mistake in the characteristic equation of the bound states, as noticed in Ref. [14]). Bound and scattering states of the $(3+1)$ Dirac equation with both vector and scalar $\delta$-shell potentials were also studied even earlier in Ref. [16]. Nevertheless, in all cited references the effects of an applied magnetic field were not discussed. A first attempt to solve the Landau quantization problem of strongly localized electrons in graphene is found in Ref. [17]. In this work, electrons were assumed to be confined in a quantum dot, where the dot was represented by a $\delta^{(2)}(\boldsymbol{r})$-potential well. Artificially representing the quantum dot by such strongly singular potential lead to divergences in the Lippmann-Schwinger equation. To overcome the problem the authors presented an approximate solution assuming that the potential of the quantum dot occupies a small but finite region and then removed the singularity. In this paper we show that the $(2+1)$ Dirac equation for the $\delta$-shell potential is free of such divergences and solve exactly the corresponding Lippmann-Schwinger equation. 


\section{Dirac equation with $\delta$-shell interaction}

The Dirac Hamiltonian in gapless graphene subjected to a perpendicular magnetic field reads

$$
\mathcal{H}=v_{F} \boldsymbol{\sigma} \cdot(\boldsymbol{p}+e \boldsymbol{A})+V_{\mathrm{imp}}(r) \equiv \mathcal{H}_{0}+V_{\mathrm{imp}}(r),
$$

where $v_{F} \sim 10^{6} \mathrm{~m} / \mathrm{s}$ is the Fermi velocity, the vector $\boldsymbol{\sigma}=\left(\sigma_{x}, \sigma_{y}\right)$ contains the Pauli matrices and the vector potential is $\boldsymbol{A}=(1 / 2) B r \boldsymbol{e}_{\theta}, \boldsymbol{e}_{\theta}$ being the polar unitary vector. Notice that the above Hamiltonian involves only one of the Dirac cones since we neglect intervalley mixing hereafter.

We are interested in the solution of the Dirac equation for the Hamiltonian (1) when the impurity potential approaches the $\delta$-shell limit. However, the resulting equation is not well defined in this limiting case. The reason lies in the fact that the product of a discontinuous function (the wave function) and the $\delta$-function is ill defined from a strict distribution theory sense. To overcome this ambiguity, we solve the Dirac equation (8) for any arbitrary sharply peaked at $r=R$ function, $R$ being the radius of the shell, and then take the $\delta$-function limit.

For the moment, we only assume that the impurity potential $V_{\text {imp }}(r)$ is axially symmetric. Then the total angular momentum, $J_{z}=L_{z}+\sigma_{z} / 2$ is conserved, and we look for eigenfunctions of the form

$$
\psi(r, \theta)=\frac{1}{\sqrt{r}}\left(\begin{array}{c}
e^{i(\kappa-1 / 2) \theta} F(r) \\
i e^{i(\kappa+1 / 2) \theta} G(r)
\end{array}\right), \quad \kappa= \pm \frac{1}{2}, \pm \frac{3}{2}, \cdots
$$

in polar coordinates. Using (1) and (2), the Dirac equation leads to

$$
\hbar v_{F}\left[i \sigma_{y} \frac{d}{d r}+\sigma_{x}\left(\frac{\kappa}{r}+\frac{e B}{2 \hbar} r\right)\right] \boldsymbol{\phi}(r)+V_{\mathrm{imp}}(r) \boldsymbol{\phi}(r)=E \boldsymbol{\phi}(r),
$$

where the upper and lower components of the radial spinor $\phi(r)$ are $F(r)$ and $G(r)$, respectively.

We introduce a dimensionless radial coordinate $z=r / l_{B}$ in terms of the magnetic length $l_{B}=\sqrt{\hbar / e B}$. The radial Dirac equation (3) is then rewritten as

$$
\left[i \sigma_{y} \frac{d}{d z}+\sigma_{x}\left(\frac{\kappa}{z}+\frac{z}{2}\right)+v_{\text {imp }}(z)\right] \phi(z)=\epsilon \phi(z),
$$

where for brevity we have defined $v_{\text {imp }}(z) \equiv V_{\text {imp }}(z) l_{B} / \hbar v_{F}$ and $\epsilon=E l_{B} / \hbar v_{F}$. After multiplying by the left by $-i \sigma_{y}$, equation (4) is solved by a Newmann solution as follows $[18]$

$$
\phi(z)=\widehat{P} \exp \left\{\int_{z_{0}}^{z} d z^{\prime}\left[\sigma_{z}\left(\frac{\kappa}{z^{\prime}}+\frac{z^{\prime}}{2}\right)+i \sigma_{y}\left(v_{\text {imp }}\left(z^{\prime}\right)-\epsilon\right)\right]\right\} \boldsymbol{\phi}\left(z_{0}\right)
$$

where $\widehat{P}$ is the ordering operator. Let $v_{\text {imp }}(z)$ be any arbitrary function of $z$ sharply peaked at $z=Z$ satisfying the limiting condition

$$
\int_{Z-\Delta Z}^{Z+\Delta Z} v_{\text {imp }}(z) d z=\lambda, \quad \Delta Z \rightarrow 0^{+}
$$


where $\lambda$ is a dimensionless coupling constant. Taking $z=Z+\Delta Z$ and $z_{0}=Z-\Delta Z$, neglecting the nonsingular terms in the integral and using the limiting condition (6) we finally obtain the following boundary condition

$$
\phi(Z+\Delta Z)=\exp \left(i \lambda \sigma_{y}\right) \phi(Z-\Delta Z),
$$

which becomes independent of how the $\delta$-function limit is taken.

\section{Landau level shift under the influence of the $\delta$-shell interaction}

The Lippmann-Schwinger solution of the dimensionless radial Dirac equation (4) is written as

$$
\phi(z)=-\lim _{\Delta Z \rightarrow 0^{+}} \int_{Z-\Delta Z}^{Z+\Delta Z} \mathcal{G}\left(z, z^{\prime} ; \epsilon\right) v_{\text {imp }}\left(z^{\prime}\right) \phi\left(z^{\prime}\right) d z^{\prime},
$$

where the Green function for the unperturbed problem is a $2 \times 2$ matrix satisfying the inhomogeneous differential equation

$$
\left[i \sigma_{y} \frac{\partial}{\partial z}+\sigma_{x}\left(\frac{\kappa}{z}+\frac{z}{2}\right)-\epsilon\right] \mathcal{G}\left(z, z^{\prime} ; E\right)=I_{2} \delta\left(z-z^{\prime}\right),
$$

where $I_{2}$ stands for the $2 \times 2$ unity matrix. The Green function exhibits a jump discontinuity at the line $z=z^{\prime}$. The value of the jump can be obtained by integration of (9) in the vicinity of this line. The result is

$$
\mathcal{G}(z+\Delta Z, z ; \epsilon)-\mathcal{G}(z-\Delta Z, z ; \epsilon)=-i \sigma_{y} .
$$

As mentioned above, the product $v_{\text {imp }}(z) \phi(z)$ is not well defined if one takes the limit $v_{\text {imp }}(z) \rightarrow \lambda \delta(z-Z)$ from the outset. Thus, we consider the same limiting procedure discussed in the previous section and solve (8) for any arbitrary sharply peaked at $z=Z$ function and then take the $\delta$-function limit.

Using the dimensionless radial Dirac equation (4) one finds that the integral equation (8) leads to (see Ref. [19] for details)

$$
\phi(z)=i \mathcal{G}(z, Z ; \epsilon) \sigma_{y}[\phi(Z+\Delta Z)-\phi(Z-\Delta Z)] .
$$

Hence we have obtained a closed expression for the perturbed eigenfunctions. The energy levels can be obtained by setting $z=Z+\Delta Z$ in (11) and using the boundary condition (7)

$$
\operatorname{det}\left[I_{2}-i \mathcal{G}(Z+\Delta Z, Z ; \epsilon) \sigma_{y}\left(I_{2}-e^{-i \sigma_{y} \lambda}\right)\right]=0 \text {. }
$$

It should be mentioned that the same condition is obtained by setting $z=Z-\Delta Z$ in (11) because of equation (10). Equation (12) determines the Landau levels perturbed by a $\delta$-shell interaction, as long as the Green function of the unperturbed problem is known. Once the energy levels have been calculated, the corresponding eigenfunctions are found by substitution of the appropriate value of $\epsilon$ into (11). 


\section{Radial Green function for Landau levels}

The radial Green function is a $2 \times 2$ matrix-valued function

$$
\mathcal{G}\left(z, z^{\prime} ; \epsilon\right)=\left(\begin{array}{ll}
G_{++}\left(z, z^{\prime} ; \epsilon\right) & G_{+-}\left(z, z^{\prime} ; \epsilon\right) \\
G_{-+}\left(z, z^{\prime} ; \epsilon\right) & G_{--}\left(z, z^{\prime} ; \epsilon\right)
\end{array}\right)
$$

satisfying the inhomogeneous differential equation (9) subjected to suitable boundary conditions. For brevity we define the differential operators $\partial_{ \pm}= \pm \partial / \partial z+\kappa / z+z / 2$. From (9) we readily obtain the off-diagonal elements of the Green function

$$
G_{ \pm \mp}\left(z, z^{\prime} ; \epsilon\right)=\frac{1}{\epsilon} \partial_{ \pm} G_{ \pm \pm}\left(z, z^{\prime} ; \epsilon\right),
$$

and also the inhomogeneous equations for the diagonal elements

$$
\frac{1}{\epsilon}\left(\partial_{ \pm} \partial_{\mp}-\epsilon^{2}\right) G_{ \pm \pm}\left(z, z^{\prime} ; \epsilon\right)=\delta\left(z-z^{\prime}\right) .
$$

Solution of the homogeneous equations can be expressed in terms of Whittaker functions, $M_{\mu \nu}$ and $W_{\mu \nu}$ [20]. Thus, the Sturm-Liouville theory leads to [21]

$$
G_{s s}\left(z, z^{\prime} ; \epsilon\right)=\frac{\epsilon}{\sqrt{z z^{\prime}}} \frac{\Gamma\left(\nu_{s}-\mu_{s}+1 / 2\right)}{\Gamma\left(2 \nu_{s}+1\right)} M_{\mu_{s} \nu_{s}}\left(\frac{z_{<}^{2}}{2}\right) W_{\mu_{s} \nu_{s}}\left(\frac{z_{>}^{2}}{2}\right),
$$

with $s= \pm$. Here $z_{>}\left(z_{<}\right)$denotes the larger (smaller) value of the pair $\left(z, z^{\prime}\right)$ and we define $\mu_{ \pm}=\left(\epsilon^{2}-\kappa \mp 1 / 2\right) / 2$ and $\nu_{ \pm}=|\kappa \mp 1 / 2| / 2$. Once the diagonal elements of the Green function are known, the off-diagonal elements are obtained from (14).

\section{Results}

Let us now turn to the results. We have summarized the most interesting ones in figures 1-3. Equation (12) allows us to calculate the Landau levels perturbed by the short-range impurity potential, as a function of the potential parameters, namely the dimensionless range $Z$ and the strength $\lambda$.

Figure 1 shows the Landau levels as a function of the dimensionless coupling constant $\lambda$, for two different values of the parameter $Z=R / l_{B}$. The energy levels increase monotonically as function of $\lambda$, as expected from a system whose average potential energy increases. A remarkable feature of the perturbed Landau levels is their $\pi$-periodicity on the coupling constant, as shown in figure 1 . This $\pi$-periodicity is also found in the energy levels of the $(1+1)$ Dirac with a $\delta$-function potential [22]. It is also worth to mention that the electron energy remains finite even if the impurity potential is rather strong. Therefore, the particle cannot fall into the center, thus avoiding an anomaly of the Coulomb potential in graphene called the supercritical Coulomb center [23].

When the impurity potential is adiabatically turned off $(\lambda \rightarrow 0)$ the energy levels reduce to the Landau levels in pristine graphene. These levels correspond to the poles of the radial Green function (16), appearing when $\nu_{s}-\mu_{s}+1 / 2=-n^{\prime}, n^{\prime}$ being a nonnegative integer, namely $\epsilon^{2}=2 n^{\prime}+|\kappa \mp 1 / 2|+\kappa \pm 1 / 2+1$. This condition can 

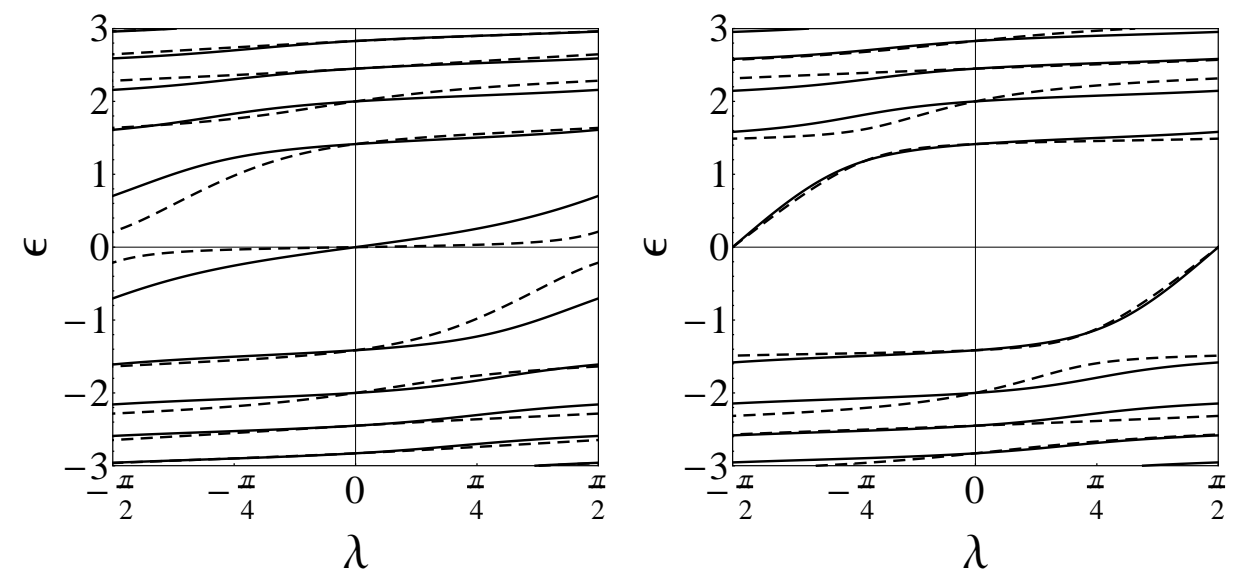

Figure 1. Shift of the dimensionless energy $\epsilon=E l_{B} / \hbar v_{F}$ of the Landau levels as a function of the dimensionless coupling constant $\lambda$. Left and right panels correspond to $\kappa=-1 / 2$ and $\kappa=1 / 2$, respectively. Solid (dashed) lines correspond to the results for $Z=0.3(Z=3)$.

be rewritten as $\epsilon= \pm \sqrt{2 n}$, with $n$ a nonnegative integer, in perfect agreement with previous results [4].

Two spatial scales are involved in the problem. In the first place, the range of the impurity potential is parameterized by the radius $R$ of the $\delta$-shell. On the other side, the magnetic length $l_{B}$ determines the spatial extent of the electronic states in the Landau level regime without impurity. Therefore, in the strong magnetic field regime $l_{B} \ll R$, namely $Z \gg 1$, one expects that the wave function is only slightly distorted by the impurity potential. Moreover, in the weak magnetic field limit $\left(l_{B} \gg R, Z \ll 1\right)$, only the wave function around the origin is affected. These two limiting situations are clearly revealed in figure 2 , which shows the evolution of the energy levels as a function of $Z$, for $\kappa=-1 / 2$ and $\lambda=\pi / 3$. As expected, the dimensionless energy approaches $\pm \sqrt{2 n}$ with $n=0,1, \ldots$ as $Z \ll 1$ or $Z \gg 1$ (dashed lines in figure 2 ). When the dimensionless coupling constant $\lambda$ is large, the energy levels display anticrossings, as shown in the inset of figure 2. Moreover, each energy level approaches the same Landau level of the unperturbed system in the two limiting situations $Z \ll 1$ and $Z \gg 1$.

In figure 3, the effect of the potential on the probability density $|\psi(r, \theta)|^{2} \sim$ $|\phi(z)|^{2} / z$ is shown for the four different values of the dimensionless range $Z$ indicated in figure 2 by solid circles. When $Z \ll 1$ or $Z \gg 1$ [panels a) and d) respectively] the wave functions are rather similar except around the origin. On the contrary, when $Z \sim 1$ $[$ panels b) and c)] the wave function is strongly affected around $z=Z$, as expected.

\section{Conclusions}

We have proved that effects of $\delta$-shell potentials on massless Dirac electrons in graphene under strong magnetic fields can be exactly evaluated using a Green function technique. The method is independent on how the $\delta$-function is taken and consequently it is free of 


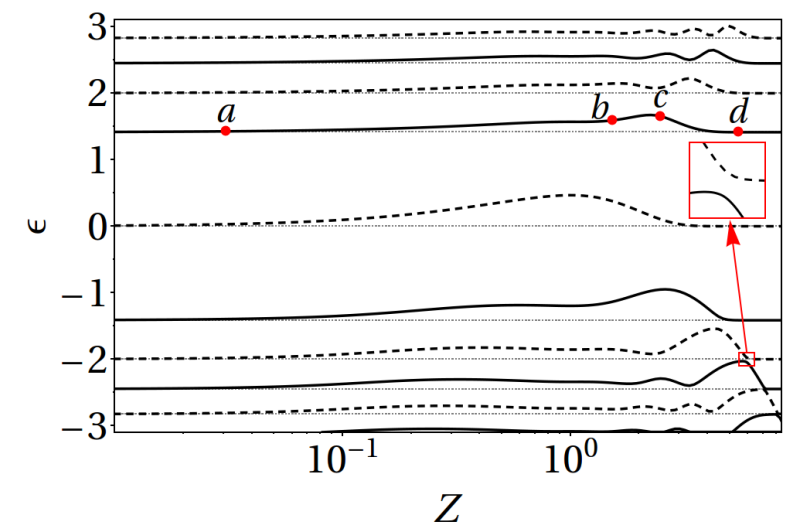

Figure 2. Shift of the dimensionless energy $\epsilon=E l_{B} / \hbar v_{F}$ of the Landau levels as a function of the dimensionless radius $Z$, for $\kappa=-1 / 2$ and $\lambda=\pi / 5$. Dotted lines represent the Landau levels of the unperturbed system. The wave functions at points marked $a-d$ are shown in figure 3 below.
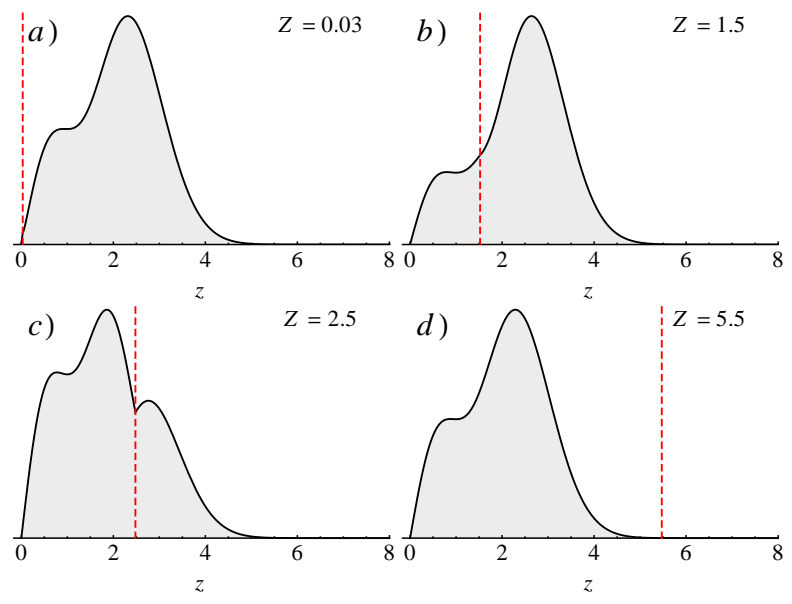

Figure 3. Plots of $|\phi(z)|^{2} / z$ as a function of the dimensionless radial coordinate $z=r / l_{B}$ for the four different values of the dimensionless range $Z$ indicated in figure 2 by circles, when $\kappa=-1 / 2$ and $\lambda=\pi / 5$.

the ambiguities appearing in defining relativistic $\delta$-interactions [18]. In particular, there is no need to regularize the potential in the Lippmann-Schwinger equation, contrary to what happens in the case of the $\delta^{(2)}(\boldsymbol{r})$-potential well [17]. In addition, the $\delta$-shell potential is free of the supercritical effects found in Coulomb potentials [23]. Therefore, $\delta$-shell potentials are good candidates to successfully replace actual and more complex impurity potentials in graphene.

\section{Acknowledgments}

This work was supported by MICINN (projects Mosaico and MAT2010-17180). The authors thank A. V. Malyshev for helpful comments. 
[1] Novoselov K S, Geim A K, Morozov S M, Jiang D, Zhang Y, Dubonos S V, Grigorieva I V and Firsov A A 2004 Science 306666

[2] Novoselov K S, Jiang D, Schedin F, Booth T J, Khotkevich V V, Morozov S M and Geim A K 2005 PNAS 10210451

[3] Wallace P R 1947 Phys. Rev. 71622

[4] Castro Neto A H, Guinea F, Peres N M R, Novoselov K S and Geim A K 2009 Rev. Mod. Phys. 81109

[5] Zhou S Y, Gweon G H, Fedorov A V, First P N, de Heer W A, Lee D H, Guinea F, Castro Neto A H and Lanzara A, Nature Mater. 20076770

[6] Domínguez-Adame F and González M A 1990 Eur. Phys. Lett. 13193

[7] González J, Guinea F and Vozmediano M A H 1994 Nucl. Phys. B 424595

[8] Novoselov K S, Geim A K, Morozov S V, Jiang D, Katsnelson M I, Grigorieva I V, Dubonos S V and Firsov A A 2005 Nature 438197

[9] Zhang Y, Tan Y W, Stormer H L and Kim P 2005 Nature 438201

[10] Du X, Skachko I, Duerr F, Luican A and Andrei E Y, 2009 Nature 462192

[11] Bolotin K I, Ghahari F, Shulman M D, Stormer H L and Kim P 2009 Nature 462196

[12] Ho J H, Lai Y H, Chiu Y H and Lin M F 2008 Physica E 401722

[13] Libisch F, Rotter S, Güttinger J, Stampfer C and Burgdörfer J 2010 Phys. Rev. B 81245411

[14] Firsova N E, Ktitorov S A and Pogorelov P A 2009 Phys. Lett. A 373525

[15] Dong S H and Ma Z Q 2002 Found. Phys. Lett. 15171

[16] Domínguez-Adame F 1990 J. Phys. A: Math. Gen. 231993

[17] Horing N J M and Liu S Y 2009 J. Phys. A: Math. Gen. 42225301

[18] McKellar B H J and Stephenson G J 1987 Phys. Rev. A 362566

[19] Méndez B and Domínguez-Adame F 1992 J. Phys. A: Math. Gen. 252065

[20] Abramowitz M and Stegun I Handbook of Mathematical Functions (Dover, New York, 1972).

[21] Duffy D G Green's Functions with Applications (Chapman \& Hall, London, 2001).

[22] Domínguez-Adame F and Maciá E 1989 J. Phys. A: Math. Gen. 22 L419

[23] Shytov A V, Katsnelson M I and Levitov LS 2007 Phys. Rev. B 99236801 